International Journal of Instrumentation and Control Systems (IJICS) Vol.2, No.2, April 2012

\title{
FAULT DETECTION AND ISOLATION USING UNKNOWN INPUT OBSERVERS WITH STRUCTURED RESIDUAL GENERATION
}

\author{
Mohammad Hossein Sobhani ${ }^{1}$ and Javad Poshtan ${ }^{2, *}$ \\ ${ }^{1}$ Department of Electrical Engineering, Iran University of Science and Technology, \\ Tehran, Iran \\ hossein.sobhani@gmail.com \\ ${ }^{2}$ Department of Electrical Engineering, Iran University of Science and Technology, \\ Tehran, Iran \\ jposhtaneiust.ac.ir
}

\begin{abstract}
Detection and isolation of faults are important tasks for improving the reliability of process industries in order to enhance their products quality. This paper investigates the detection and isolation of faults using structured residuals. Actuator and sensor faults are considered. Residuals are generated using a bank of unknown input observers (UIO). Three-tank benchmark system was used as a prototype of many process industries. Simulation results show the effectiveness of the studied method.
\end{abstract}

\section{KEYWORDS}

Fault detection and isolation, Three-tank benchmark, Residual generation, Residual evaluation, Unknown input observer

\section{INTRODUCTION}

Early detection and isolation of faults are critical tasks in modern process industries. Many research works have been made during last decades to improve fault detection and isolation methods. Extensive reviews of different fault detection and isolation methods can be found in the literature [1-7]. Existing methods can be grouped into three general categories: quantitative model-based methods, qualitative model-based methods and data driven methods. Quantitative model-based methods have received considerable attention in recent years [8]. These approaches use the mathematical model of the process to estimate its normal behaviour. Differences between the estimated and the actual behaviour are symptoms or fault indicators. These differences are called residuals. Later, the residuals are evaluated aiming at to localize the fault. Although there is a close relationship among the quantitative model-based techniques, observer-based approach have become the most popular and important method for model-based fault detection and isolation $[9,10]$, especially within the automatic control community.

\footnotetext{
* Corresponding author

DOI : $10.5121 /$ ijics.2012.2201
} 
Fault isolation can be achieved by generating structured residuals. These structured residuals can be generated using different methods. One of the best methods for generating structured residuals is to use unknown input observers (UIO). These observers are designed such that they are insensitive to certain faults while are sensitive to other faults in the system. Using a bank of well designed UIOs, faults can be detected and isolated simply with the help of the generated residuals.

Three-tank benchmark system can be viewed as a good prototype of many industrial applications in process industry, such as chemical and petrochemical plants, oil and gas systems [11]. In this paper fault detection and isolation of the three-tank benchmark system with structured residual generation using UIOs is studied.

\section{THREE-TANK BENCHMARK SYSTEM}

The three-tank benchmark system has many similarities to various chemical and petrochemical processes and though is a good prototype for testing different fault detection and isolation methods in order to evaluate their performances. A simple schematic for this benchmark system is shown in Figure 1. As it can be seen this system is made of three cylindrical tanks which are named, from left to right, as T1, T3, and T2, respectively. These tanks are connected serially to each other with cylindrical pipes. T2 has an outflow with another cylindrical pipe. The outflow coefficients of these pipes are named as $\mu_{13}, \mu_{32}$, and $\mu_{20}$, from left to right respectively.

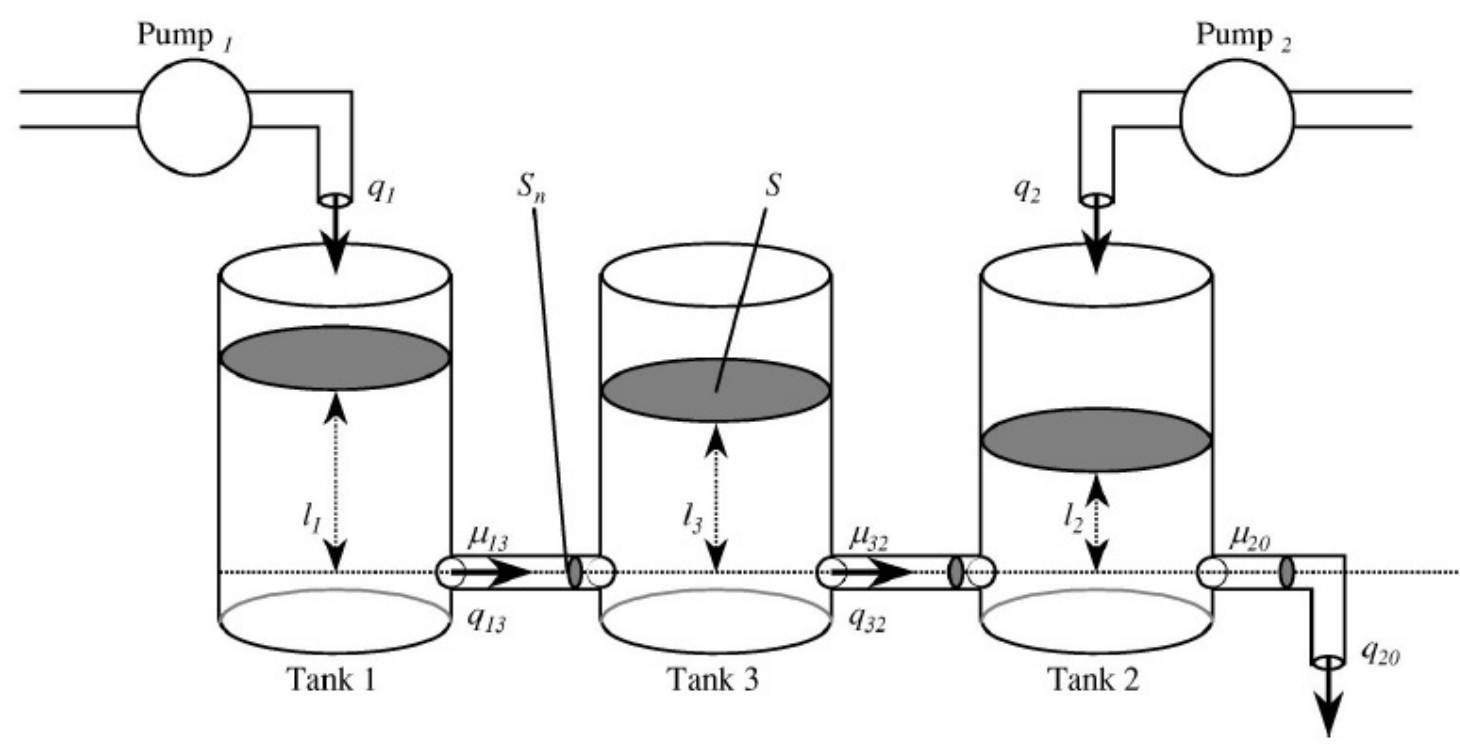

The typical control issue involved in this system is how to keep the desired water levels in the ending tanks (T1 and T2). The manipulated control inputs are the flow rates of the two pumps named $\mathrm{P} 1$ and $\mathrm{P} 2$, and are respectively pumping water in $\mathrm{T} 1$ and $\mathrm{T} 2$. The flow rates of these pumps are controlled via their voltage inputs. $q_{1}$ and $q_{2}$ are respectively P1 and P2 flow rates and $l_{1}, l_{2}$, and $l_{3}$ are water levels in $\mathrm{T} 1, \mathrm{~T} 2$, and $\mathrm{T} 3$ respectively. The constraints of the system include the pumps maximum flow rates and maximum level of water in each tank. Each tank is 
equipped with a pressure sensor which gives a voltage output proportional to water level in that tank and each pump output flow rate is measured by a flow meter.

Writing the mass balance equations we will get:

$S \frac{d l_{1}(t)}{d t}=q_{1}(t)-q_{13}(t)$

$S \frac{d l_{2}(t)}{d t}=q_{2}(t)+q_{32}(t)-q_{20}(t)$

$S \frac{d l_{3}(t)}{d t}=q_{13}(t)-q_{32}(t)$

Where $S$ is the cross sectional area of the tanks, $q_{13}$ and $q_{32}$ are the flow rates between T1 and $\mathrm{T} 3$, and $\mathrm{T} 3$ and $\mathrm{T} 2$, respectively, and $q_{20}$ is the outflow from $\mathrm{T} 2$. These flow rates are not measured but can be computed using the Torriceli-rule:

$$
\begin{aligned}
& q_{13}(t)=\mu_{13} S n \times \operatorname{sgn}\left[l_{1}(t)-l_{3}(t)\right] \times \sqrt{2 g\left|l_{1}(t)-l_{3}(t)\right|} \\
& q_{32}(t)=\mu_{32} S n \times \operatorname{sgn}\left[l_{3}(t)-l_{2}(t)\right] \times \sqrt{2 g\left|l_{3}(t)-l_{2}(t)\right|} \\
& q_{20}(t)=\mu_{20} S n \times \sqrt{2 g l_{2}(t)}
\end{aligned}
$$

Where $S n$ is the cross sectional area of the connecting pipes and $g$ is the earth gravity constant. Using equations (1) and (2) we can write the nonlinear state space model of the system as follows:

$$
\begin{aligned}
& \frac{d x(t)}{d t}=f(x(t), u(t)) \\
& y(t)=x(t)
\end{aligned}
$$

Where $y(t)=x(t)=\left[\begin{array}{lll}l_{1} & l_{2} & l_{3}\end{array}\right]^{T}$ and $u(t)=\left[\begin{array}{ll}q_{1} & q_{2}\end{array}\right]^{T}$.

\section{UNKNOWN INPUT OBSERVERS}

Using unknown input observers (UIO) is one of the best ways to generate structured residuals for fault detection and isolation [12]. This class of observers are designed so that they are insensitive to some certain disturbances which we know how they affect the system but we do not know the disturbances themselves. This way UIOs are best suitable for generation of robust structured residuals in order to detect and isolate faults.

The UIO is a generalization of the Luenberger observer [13] which is used here for fault detection and isolation.

Considering that the fault may occur because of the failure of the system equipments such as sensors and actuators, or some unknown disturbances, the state space representation of the system can be written as follows: 


$$
\begin{aligned}
& x(k+1)=A x(k)+B u(k)+E f(k) \\
& y(k)=C x(k)
\end{aligned}
$$

Where $f$ is the fault vector (including disturbances) and $E$ is the fault distribution matrix. $E$ is a known matrix. To isolate the fault, the fault vector is divided into two parts as $f=\left[\begin{array}{ll}f_{1} & f_{2}\end{array}\right]^{T}$. $f_{1}$ contains the faults which the observer is not sensitive to and $f_{2}$ contains the faults which the observer is sensitive to. Also matrix $E$ is divided into $E=\left[\begin{array}{ll}E_{1} & E_{2}\end{array}\right]$ as well.

An UIO structure is as follows:

$$
\begin{aligned}
& z(k+1)=F z(k)+T B u(k)+K_{12} y(k) \\
& \hat{x}(k)=z(k)+H y(k)
\end{aligned}
$$

Where $z$ is the UIO state vector which is computed by linear transformation $z(k)=T x(k)$ and $\hat{x}$ is the estimated state vector of the system. $F, T, H$, and $K_{12}$ are matrices that will be designed such that the unknown input $\left(f_{1}\right)$ will be decoupled from other inputs. For this purpose certain design requirements will be attended. The state estimation error $\left(e_{x}=x-\hat{x}\right)$ and the residual are defined by:

$$
\begin{aligned}
& e_{x}(k+1)=\left(A-H C A-K_{1} C\right) e_{x}(k) \\
&+\left(\left(A-H C A-K_{1} C\right)-F\right) z(k) \\
&+((I-H C)-T) B u(k) \\
&+\left(\left(A-H C A-K_{1} C\right) H-K_{2}\right) y(k) \\
&+(I-H C) E_{1} f_{1}(k)+(I-H C) E_{2} f_{2}(k) \\
& r(k)=y(k)-C \hat{x}(k)=(I-H C) y(k)-C z(k)
\end{aligned}
$$

If the following equations hold:

$$
T=I-H C, \quad T E_{1}=0, \quad F=T A-K_{1} C, \quad K_{2}=F H, \quad K_{12}=K_{1}+K_{2}
$$

Then equation (6) will become:

$$
e_{x}(k+1)=F e_{x}(k)+T E_{2} f_{2}(k)
$$

So as it can be seen if the eigenvalues of $F$ are stable and $f_{2}=0$, regardless of what $f_{1}$ is, the state estimation error will reach zero asymptotically. This means that the observer is insensitive to the unknown input $f_{2}$.

Assuming that the equations defined in (8) hold true, a specific solution for the UIO design is to determine $H$ as follows [13]:

$$
H=E_{1}\left(C E_{1}\right)^{+}
$$


Where $\left({ }^{+}\right)$denotes the Moore-Penrose pseudo-inverse.

According to [14] the necessary and sufficient conditions for the existence of a full-order UIO for the system defined by (4) are:

1. $\operatorname{rank}\left(C E_{1}\right)=\operatorname{rank}\left(E_{1}\right)$

2. $(C, T A)$ is a detectable pair.

\section{FAUlt DETECTION AND ISOlATION}

In order to detect and isolate faults in a system we need to look for some fault symptoms. The most common fault symptom that is used for fault detection and isolation is residual. The common procedure for fault detection and isolation using residuals is made of two main steps: residual generation, and residual evaluation.

\subsection{Residual Generation}

Residual generation is the core element of an observer based fault detection system. The residuals are generated using the difference between the real and the estimated output of the system. This difference is usually computed using the norm of the output estimation error vector.

UIO approach for fault detection makes use of the disturbance decoupling principle, in which the residual is computed assuming the decoupling of the effects of faults on different inputs. For the purpose of fault isolation, it is also assumed that the effect of a fault is decoupled from the effects of other faults.

A well designed residual signal is defined such that it is equal or near zero in the fault free case and is clear from zero when the system is faulty.

$$
\begin{array}{lll}
r(t)=0 \quad(\text { or } \quad r(t) \approx 0) & & \text { Fault free case }, \\
r(t) \neq 0 & & \text { Faulty case }
\end{array}
$$

\subsection{Residual Evaluation}

The residual is examined in terms of the likelihood of a fault, and a logical decision-making process is then applied aiming at to decide if the fault has occurred and avoid wrong decisions, such as false alarm and fault ignored [15]. The final decision is made after a simple comparison between a threshold $T(t)$ (it can be adaptive or constant) and the residual evaluation function $J(r(t))$. This decision is shown by a binary variable $S_{r}$. This binary variable is set to 1 if the value of the residual evaluation function $J(r(t))$ exceeds the threshold $T(t)$ value, otherwise it is set to 0 .

$$
\begin{aligned}
& J(r(t)) \leq T(t) \text { then } S_{r}=0 \quad \text { (Fault free case), } \\
& J(r(t))>T(t) \text { then } S_{r}=1 \quad \text { (Faulty case) }
\end{aligned}
$$




\subsection{Fault Detection and Isolation with UIOs}

To use UIOs for fault detection and isolation, the system have to be described in a way that suits for UIO designing process. For this purpose the system is linearized around the operating point $u_{o}=\left[\begin{array}{ll}3.5 & 3.18\end{array}\right]^{T} \times 10^{-5}$ and $y_{o}=\left[\begin{array}{lll}0.4 & 0.2 & 0.3\end{array}\right]^{T}$, and then discretized with sampling time $T=1$. After linearization and discretization of the system the faults can be defined in the system as follows:

$$
\begin{aligned}
& x(k+1)=A x(k)+B u(k)+E_{a} f_{a}(k) \\
& y(k)=C x(k)+E_{s} f_{s}(k)
\end{aligned}
$$

Where $f_{a}$ and $f_{s}$ are actuators and sensors fault vectors, respectively. Also $E_{a}$ and $E_{s}$ are the actuators and sensors fault distribution matrices, respectively. Fault vectors $f_{a}$ and $f_{s}$ are unknown but their distribution matrices $E_{a}$ and $E_{s}$ are known matrices. In this work these matrices are defined as follows:

$$
\begin{aligned}
& E_{a}=B, \\
& E_{s}=C
\end{aligned}
$$

This definition is due to the nature of the actuators and sensors faults which have same effects on the system as inputs and measurements, respectively. Also the matrices $A, B$, and $C$ are computed as follow after linearization and discretization of the continuous nonlinear system:

$$
A=\left[\begin{array}{ccc}
0.9887 & 0 & 0.0112 \\
0 & 0.9737 & 0.0112 \\
0.0078 & 0.0078 & 0.9841
\end{array}\right], \quad B=\left[\begin{array}{cc}
64.57 & 0.001 \\
0.001 & 64.08 \\
0.2574 & 0.2561
\end{array}\right], \quad C=I_{3}
$$

To design UIOs the system should be represented in the appropriate form as mentioned in (4). For actuator faults there is no need for any change in the system representation. Assuming that there will not be more than one fault at a time, the system can be represented as follows:

$$
\begin{aligned}
& x(k+1)=A x(k)+B u(k)+E_{a} f_{a}(k) \\
& y(k)=C x(k)
\end{aligned}
$$

An UIO will be designed for any of the actuators. For the $i$ th actuator the system will be rewritten as follows:

$$
\begin{aligned}
& x(k+1)=A x(k)+B u(k)+E_{a, i} f_{a, i}(k)+\bar{E}_{a, i} \bar{f}_{a, i}(k) \\
& y(k)=C x(k)
\end{aligned}
$$


Where $f_{a, i}$ is the $i$ th row of $f_{a}$ which shows the $i$ th actuator fault that is supposed to be the unknown input, and $E_{a, i}$ is the $i$ th column of $E_{a}$. Also $\bar{f}_{a, i}$ and $\bar{E}_{a, i}$ are actuator vector fault and actuator fault distribution matrix without their $i$ th row and column, respectively.

Similar to actuator faults case, again with the assumption of the occurrence of only one fault at a time, the system can be rewritten as follows for sensor faults:

$$
\begin{aligned}
& x(k+1)=A x(k)+B u(k) \\
& y(k)=C x(k)+E_{s, j} f_{s}(k)+\bar{E}_{s, j} \bar{f}_{s, j}(k)
\end{aligned}
$$

Where $f_{s, i}$ is the $i$ th row of $f_{s}$ which shows the $i$ th sensor fault, and $E_{s, i}$ is the $i$ th column of $E_{s}$. Also $\bar{f}_{s, i}$ and $\bar{E}_{s, i}$ are sensor vector fault and sensor fault distribution matrix without their $i$ th row and column, respectively. As it can be seen this representation of system is not similar to (4) that is appropriate for the UIO design and a change is needed to make the representation of the system suitable for UIO design.

According to [16], a system affected by a sensor fault can be written as a system represented by an actuator fault. Assume the dynamic of a sensor fault described as:

$$
f_{s, j}(k+1)=f_{s, j}(k)+T \xi(k)
$$

Where $\xi$ defines the sensor error input and $T$ is the sampling time which is here equal to $1 \mathrm{~s}$.

From (18) and (19), a new system representation including this auxiliary state can be introduced:

$$
\begin{aligned}
& {\left[\begin{array}{c}
x(k+1) \\
f_{s, j}(k+1)
\end{array}\right]=\left[\begin{array}{cc}
A & 0_{n \times 1} \\
0_{1 \times n} & 1
\end{array}\right]\left[\begin{array}{c}
x(k) \\
f_{s, j}(k)
\end{array}\right]+\left[\begin{array}{c}
B \\
0
\end{array}\right] u(k)+\left[\begin{array}{c}
0_{n \times 1} \\
T
\end{array}\right] \xi(k),} \\
& y(k)=\left[\begin{array}{ll}
C & E_{s, j}
\end{array}\right]\left[\begin{array}{c}
x(k) \\
f_{s, j}(k)
\end{array}\right]
\end{aligned}
$$

This representation is now suitable for UIO design.

A bank including 5 UIOs (2 UIOs for actuators and 3 UIOs for sensors) were designed to detect and isolate sensor and actuator faults. Each UIO is designed to be insensitive to certain fault so that its output does not change when that fault occurs. When a fault occurs all the UIOs will be affected except the one that is insensitive to that fault and this will help us to detect and isolate the fault.

But when a fault occurs in T1 sensor or actuator, both UIOs which are designed to be insensitive to T1 actuator fault and to the T1 sensor fault are unaffected and we cannot tell where the fault has occurred. This problem also exists for T2 actuator and sensor. This is because of the similarity between the UIOs designed for actuator and sensor of the same tanks. To solve this problem a $6^{\text {th }}$ UIO were designed such that it is insensitive to both actuators faults. This additional UIO will tell us whether the sensor or the actuator is faulty. 


\section{Simulation RESUltS}

Results of three fault scenarios are presented here. Other possible faults are similar to the ones presented here and does not bring any new insight into the application of method and so are not included here. In all cases presented here, the process is assumed to be initially at steady state. Also the fault free case residuals are shown in Figure 2.

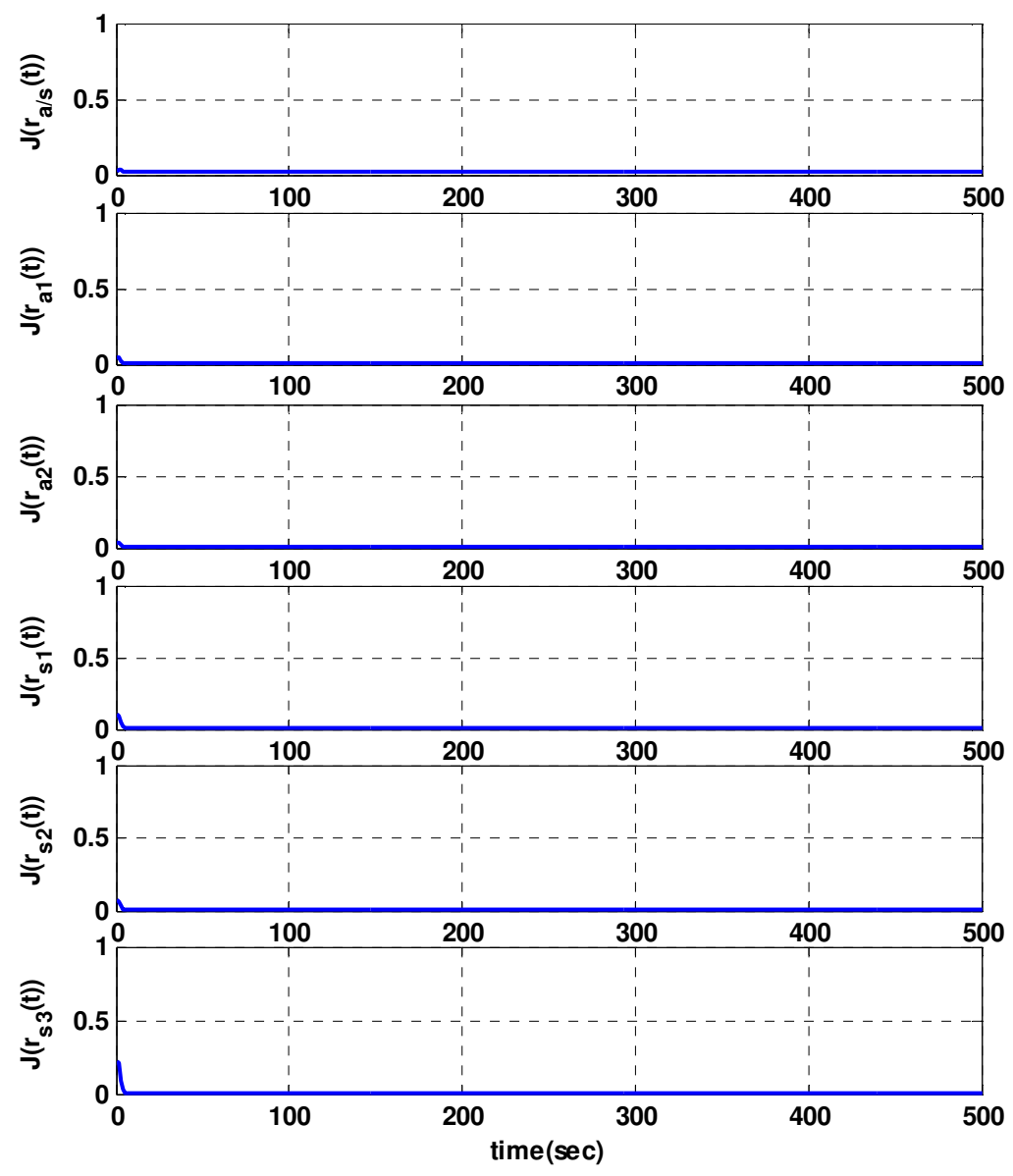

Figure 2. Fault free case residuals

As it can be seen in Figure 2, the residuals are near zero when there is no fault in the system.

\subsection{Fault Scenario I}

A sudden decrease of $80 \%$ gain occurs in pomp 2 at $t=200$. Residuals can be seen in Figure 3. It can be seen that $J\left(r_{a / s}(t)\right)$ has not changed and it shows that the system is wheather not faulty or it has a fault in actuators. $J\left(r_{a 1}(t)\right)$ has increased at $t=200$ but $J\left(r_{a 2}(t)\right)$ has not been affected and this shows that a fault has occurred in actuator 2 at $t=200$. 


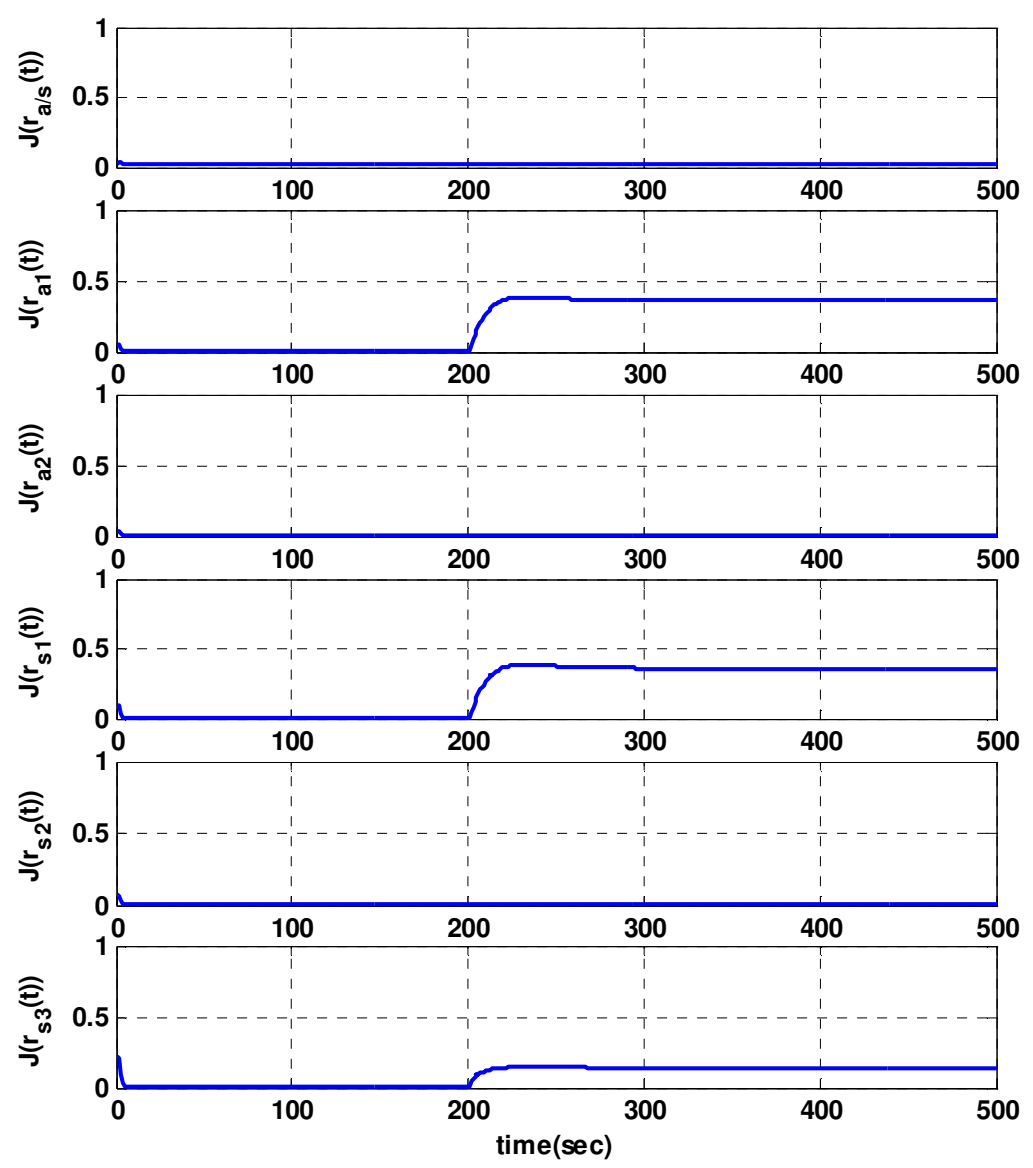

Figure 3. Residuals for fault scenario I

\subsection{Fault Scenario II}

A sudden decrease of $50 \%$ gain occurs in sensor 1 at $t=200$. Residuals can be seen in Figure 4 . Figure 3 shows that $J\left(r_{a / s}(t)\right)$ has a change at $t=200$. This means that a fault has occurred in sensors. Also $J\left(r_{s 2}(t)\right)$ and $J\left(r_{s 3}(t)\right)$ have a change at $t=200$ but $J\left(r_{s 1}(t)\right)$ is unaffected and this shows that the fault has occurred in sensor 1 . 


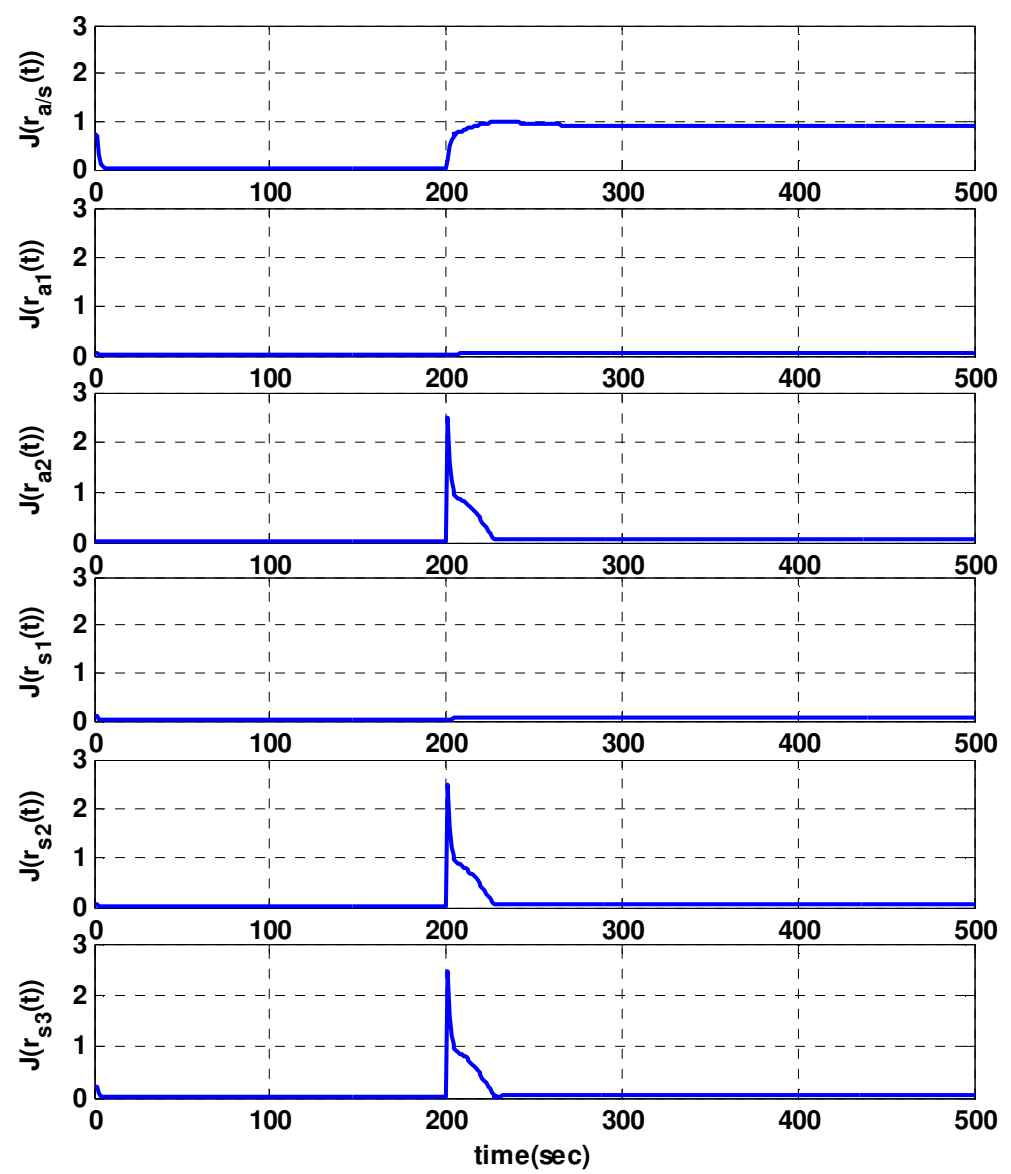

Figure 4. Residuals for fault scenario II

\subsection{Fault Scenario III}

At $t=200$, a constant value $e=0.05$, is added to the output of sensor 2. Residuals can be seen in Figure 5.

Figure 4 shows that $J\left(r_{a / s}(t)\right)$ has a change at $t=200$. This means that a fault has occurred in sensors. Also $J\left(r_{s 1}(t)\right)$ and $J\left(r_{s 3}(t)\right)$ have a change at $t=200$ but $J\left(r_{s 2}(t)\right)$ is unaffected and this shows that the fault has occurred in sensor 2 . 


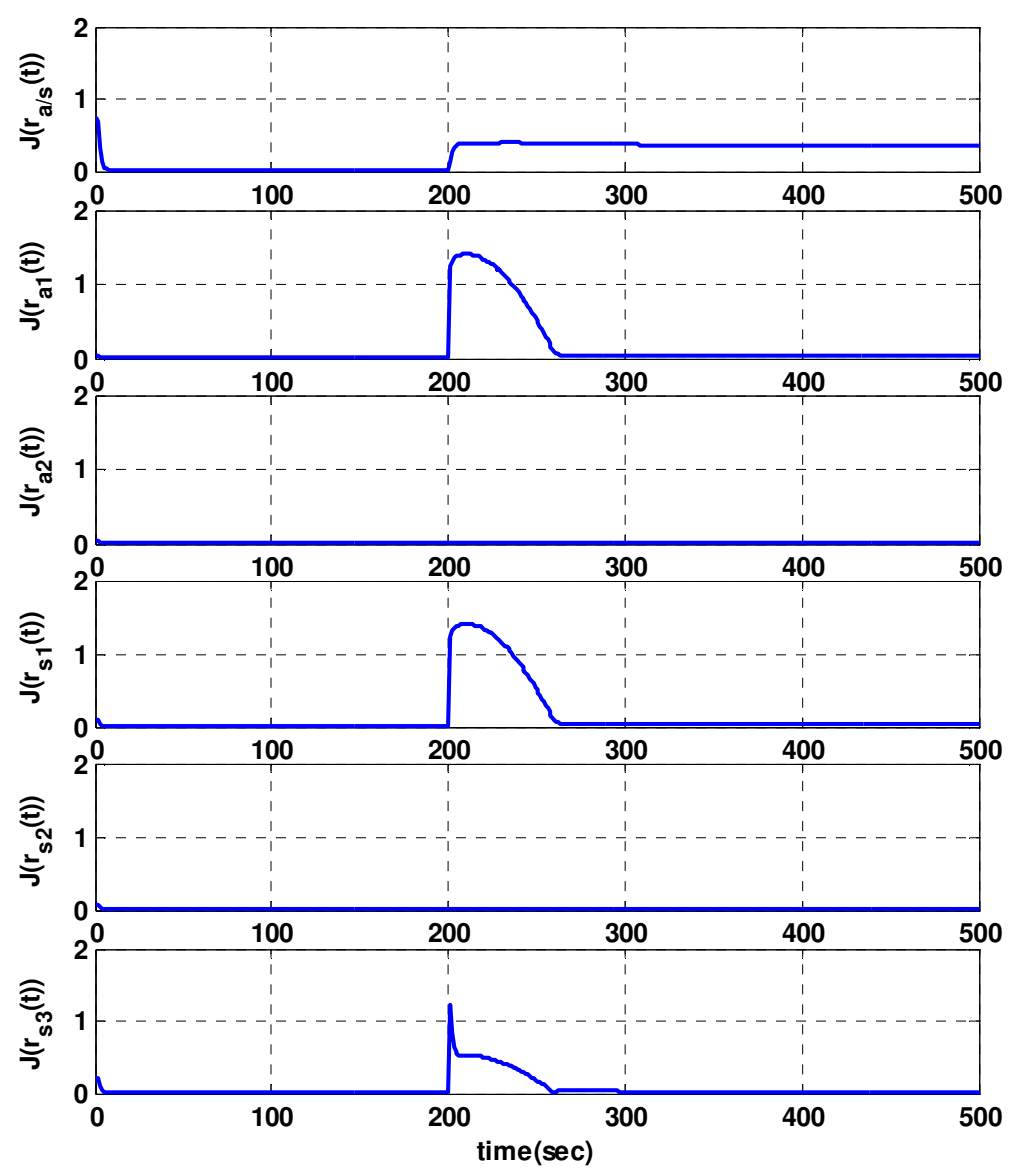

Figure 5. Residuals for fault scenario III

\section{CONCluSions}

A fault detection and isolation scheme based on structured residual generation has been studied to detect and isolate sensor and actuator faults in three tank benchmark system. A bank of UIOs was used to generate structured residuals for isolation of faults. As the faults of the sensor and actuator of the same tank could not be distinguished a $6^{\text {th }}$ UIO was added to the bank that both actuators faults were its unknown inputs, so this $6^{\text {th }}$ UIO could tell us if any sensor is faulty or not, and helped us in isolation of faults. Three fault scenarios were studied: actuator gain deterioration, sensor gain deterioration and sensor bias. Simulation results show the good performance of the fault detection and isolation system. However, this fault detection and isolation scheme cannot isolate simultaneous faults, and this is the open research area for development of this method. 
International Journal of Instrumentation and Control Systems (IJICS) Vol.2, No.2, April 2012

\section{REFERENCES}

[1] A.S. Willsky, "A survey of design methods for failure detection in dynamic systems", Automatica 12 (6) (1976) pp. 601-611

[2] J.J. Gertler, "A survey of model-based failure detection and isolation in complex plants", IEEE control system magazine 8 (6) (1988) pp. 3-11

[3] P.M. Frank, "Fault diagnosis in dynamic systems using analytical and knowledge-based redundancyA survey and some new results", Automatica 26 (3) (1997) pp. 459-474

[4] R.J. Patton, "Robustness in model-based fault diagnosis: the 1995 situation", Annual Reviews in Control 21 (1) (1997) pp. 103-123

[5] R. Isermann, "Supervision, fault-detection and fault-diagnosis methods-An introduction", Control Engineering Practice 5 (5) (1997) pp. 639-652

[6] P.M. Frank, S.X. Ding, T. Marcu, "Model-based fault diagnosis in technical processes", Tran. Inst. Measure. Contr. 22 (1) (2000) pp. 57-101

[7] V. Vankatasubramanian, R. Rengaswamy, K. Yin, S.N. Kavuri, "A review of process fault detection and diagnosis. Part I. quantitative model-based methods", Comput. Chem. Eng. 27 (3) (2003) pp. 293311

[8] O. A.Z. Sotomayer, D. Odloak, "Observer-based fault diagnosis in chemical plants", Chemical Engineering Journal 112 (2005) pp. 93-108

[9] R.J. Patton, J. Chen, "Observer-based fault detection and isolation: robustness and applications", Cont. Eng. Pract. 5 (5) (1997) pp. 671-682

[10] P.M. Frank, X. Ding, "Survey of robust residual generation and evaluation methods in observer-based fault detection systems", J. Process Contr. 7 (6) (1997) pp. 403-424

[11] M. Kubalcik, V. Bobal, "Adaptive control of three - tank - system: comparison of two methods", 16th Mediterranean Conference on Control and Automation Congress Centre, Ajaccio, France, June 25-27, 2008

[12] J. Chen, R. J. Patton, Robust Model-Based Fault Diagnosis for Dynamic Systems, Kluwer Academic Publishers, (1999)

[13] O. A.Z. Sotomayor, D. Odloak, "Observer-Based Fault Diagnosis in Chemical Plants", Chemical Engineering Journal, Vol. 112, pp-93-108, (2005)

[14] M. Hou, P.C. Muller, "Disturbance Decoupled Observer Design: A Unified Viewpoint", IEEE Transaction on Automatic Control, Vol. 39, No. 6, pp-1338-1341, (1994)

[15] M. H. Sobhani, J. Poshtan, "Observer-based Fault Detection and Isolation of Three-tank Benchmark System", $2^{\text {nd }}$ Conference on Control, Instrumentation ,and Automation, Shiraz, Iran, 26-28 Nov., 2011

\section{Mohammad Hossein Sobhani}

He was born in iran. He got his B.Sc. degree from Shiraz University and now is M.Sc. student at Iran University of Science and Technology. His research area is process control and fault detection and isolation.

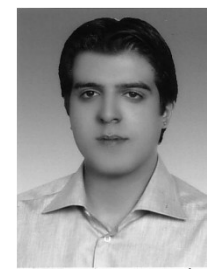

\section{Javad Poshtan}

He received his BSc, MSc and $\mathrm{PhD}$ degrees in Electrical Engineering from Tehran University, Tehran, Iran, in 1987; Sharif University of Technology, Tehran, Iran, in 1991; and University of New Brunswick, Canada, in 1997, respectively. Since 1997 he has been with the Department of Electrical Engineering at Iran University of Science and Technology. He is involved in academic and research activity in areas such as control system theory, system identification, estimation theory, and fault diagnosis.

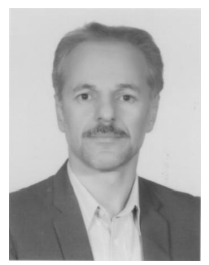

